\title{
Effect of Low-level Laser on LI4 Acupoint in Pain Reduction during Local Anesthesia in Children
}

\author{
Sandhyarani $\mathrm{B}^{1}$, Ramoli R Pawar ${ }^{2}$, Anil T Patil $^{3}$, Mital V Kevadia ${ }^{4}$
}

\begin{abstract}
Background: Pain is a multidimensional construct that involves sensory, emotional, and cognitive processes. It is an essential component of child behavior guidance. The injection of a local anesthetic agent during pediatric dental treatment is one of the most painful and distressing procedures performed, stimulation of acupoint LI4 provides an analgesic effect in the orofacial region, thus decreasing the pain during injection.

Aims and objectives: To compare and evaluate the effect of low-level laser on LI4 acupoint and surface-acting $20 \%$ benzocaine gel during local anesthesia.

Materials and methods: Children of age-group between 5 years and 9 years receiving bilateral local anesthesia were scheduled for dental treatment. Split-mouth cross-over study was planned and was divided into two groups, receiving low-level laser acupuncture on LI4 acupoint with placebo as a moist cotton swab in the first visit and $20 \%$ benzocaine gel with placebo as low-level laser acupuncture off mode in second visit and vice versa. Pain intensity was evaluated using the sound eye motor scale as subjective scale, Wong-Bakers pain rating scale. Pulse rate was measured before, during, and after the procedure using a pulse oximeter.

Results: The average heart rate, Wong-Bakers pain rating scale, and Sound Eye Motor scale were significantly lower in the group having lowlevel laser when compared with the group having placebo low-level laser therapy.

Conclusion: The low-level laser can be used to control pain during local anesthesia in children.

Keywords: Acupuncture, Laser acupuncture, Local anesthesia, Low-level laser therapy.

International Journal of Clinical Pediatric Dentistry (2021): 10.5005/jp-journals-10005-1995
\end{abstract}

\section{INTRODUCTION}

Cooperation of children by using proper pain control methods during a dental procedure is a challenge in pediatric dentistry. The American Academy of Pediatric Dentistry (AAPD) says that children may vary in their cognitive and emotional development and their pain responses. ${ }^{1}$ Dental treatment involves an injection of local anesthesia which is painful and distressing procedures performed on children. ${ }^{2,3}$ Currently, there have been various methods introduced to reduce the pain during injection of local anesthesia. ${ }^{4,5}$ However, nowadays, acupuncture is used as an alternative therapeutic procedure to control pain in dentistry, including facial pain and dental pain. ${ }^{6,7}$

Acupuncture originated from traditional Chinese medicine which says that the body is divided into various meridians and pain is caused due to stagnation of Qi also when certain points along this meridian are stimulated it allows free flow of Qi. ${ }^{8} \mathrm{LI} 4$ point showed greater analgesic effect when compared with placebo or shame control procedures in both experimental and clinical settings. ${ }^{8,9}$ However, use of traditional needles for acupuncture in children can be fearful and can not be accepted by the parents, therefore laser acupuncture is appropriate for treating children and adults for stimulating points of difficult access. Laser acupuncture is defined as stimulation of these traditional acupuncture points using a low-level laser which is not only painless but also noninvasive, atraumatic, and easy to perform, and no risk of cross infections. ${ }^{10}$

Nowadays, studies have been dated which demonstrate the effectiveness of laser on acupuncture points for various dental procedures like myofascial pain, gag reflex, and temporomandibular dysfunction. ${ }^{10}$ However, no literature is available to stimulate acupuncture points using low-level laser therapy for pain control
${ }^{1-4}$ Department of Pediatric and Preventive Dentistry, Bharati Vidyapeeth Dental College and Hospital, Sangli, Maharashtra, India

Corresponding Author: Ramoli R Pawar, Department of Pediatric and Preventive Dentistry, Bharati Vidyapeeth Dental College and Hospital, Sangli, Maharashtra, India, Phone: +91 9545080350, e-mail: ramolipawar1693@gmail.com

How to cite this article: Sandhyarani B, Pawar RR, Patil AT, et al. Effect of Low-level Laser on LI4 Acupoint in Pain Reduction during Local Anesthesia in Children. Int J Clin Pediatr Dent 2021;14(4):462-466.

Source of support: Nil

Conflict of interest: None

during local anesthesia. Thus, the objective of the present study was to evaluate the effect of low-level laser therapy on LI4 acupoint to reduce pain during local anesthesia.

\section{Materials and Methods}

The study was conducted in the Department of Pedodontics and Preventive Dentistry, Bharati Vidyapeeth Dental College and Hospital, Sangli, after the ethical clearance from the institutional review board and written consent from parents was obtained and based on inclusion and exclusion criteria.

Forty children aged between 5 and 9 years of age were enrolled in the study.

Each child's parents were explained in detail about the procedure. They were informed about their right to refuse or discontinue their participation in the study at any time and were then asked to sign the consent form. 


\section{Inclusion Criteria}

- Child's first dental visit.

- Children requiring local anesthesia bilaterally on the maxillary arch.

- Frankel behavior ratings 3 and 4 .

\section{Exclusion Criteria}

- Children with a history of allergy to lignocaine in local anesthesia,

- Children who are medically compromised and special children.

- Children with Frankel behavioral ratings 1 and 2 .

\section{Study Design}

It is a randomized crossover study and subjects were randomly divided into two groups; group I and group II. As the pain perception of the patient may vary with second visits a crossover study was planned (Flowchart 1).

\section{First Visit}

Group I: Children receiving low-level laser acupuncture at LI4 point, placebo used was moist cotton swab at the area of infiltration. Group II: Children receiving 20\% benzocaine gel at the area of infiltration, placebo used was low-level laser acupuncture on off mode at LI4 point.

\section{Second Visit}

Group I: Children receiving 20\% benzocaine gel at the area of infiltration, placebo used was low-level laser acupuncture on off mode at LI4 point.

Group II: Children receiving low-level laser acupuncture at LI4 point, placebo used was moist cotton swab in the area of infiltration.

LI4 (Hegu), an acupuncture point was selected based on traditional Chinese medicine to control dentofacial pain. ${ }^{8,9}$ This Hegu point is located on the back of the hand, between the first and second metacarpal bones, at the midpoint of the second metacarpal bone, close to its radial border. To identify this point, the patient is asked to adduct the thumb and index finger the point was located at the highest point of the first and second metacarpal bone. ${ }^{11}$

\section{During the First Visit}

In group I $(n=20)$, a moist cotton swab was applied at the area of infiltration to induce placebo and waited for 1 minute and then lowlevel laser acupuncture was done on LI4 point with a power output of $200 \mathrm{~mW}$, at a wavelength of $980 \mathrm{~nm}$, and energy of 3-4 J (Zolar photon plus diode laser) for 1 minute followed by local infiltration ( $2 \%$ lignocaine and 1:80,000 adrenaline) of $0.5 \mathrm{~mL}$ for 30 seconds on the buccal surface of the respective tooth which was given by another operator who was not aware of groups ${ }^{12,13}$ (Fig. 1).

In group II $(n=20)$, during the first visit, during the procedure, a cotton roll for 30 seconds was used to keep mucosa dry followed by the application of $20 \%$ of topical benzocaine gel for 1 minute. ${ }^{14}$ Then, low-level laser acupuncture was done on "off mode" at LI4 point to induce placebo effect followed by local infiltration ( $2 \%$

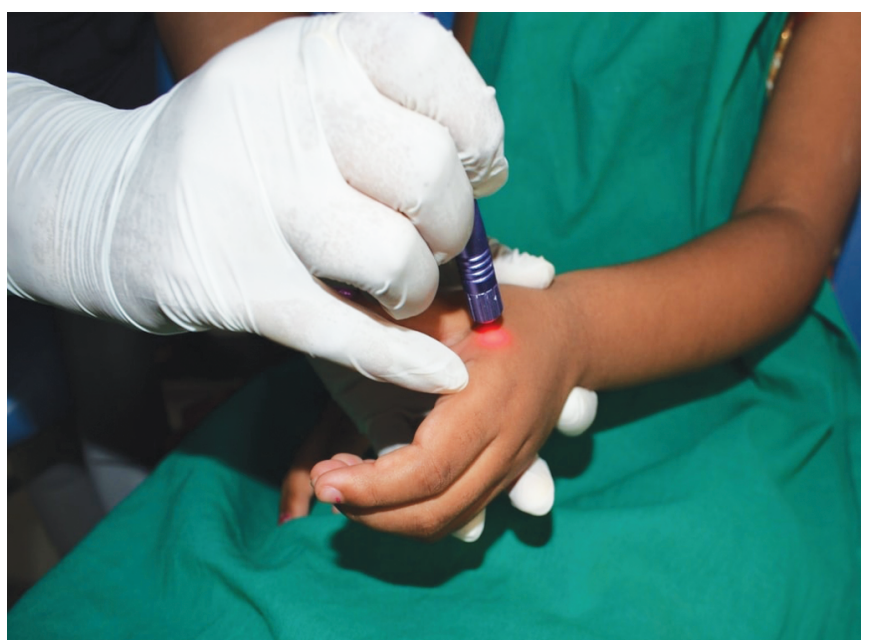

Fig. 1: Low-level laser acupuncture on LI4 acupoint

Flowchart 1: Crossover study design

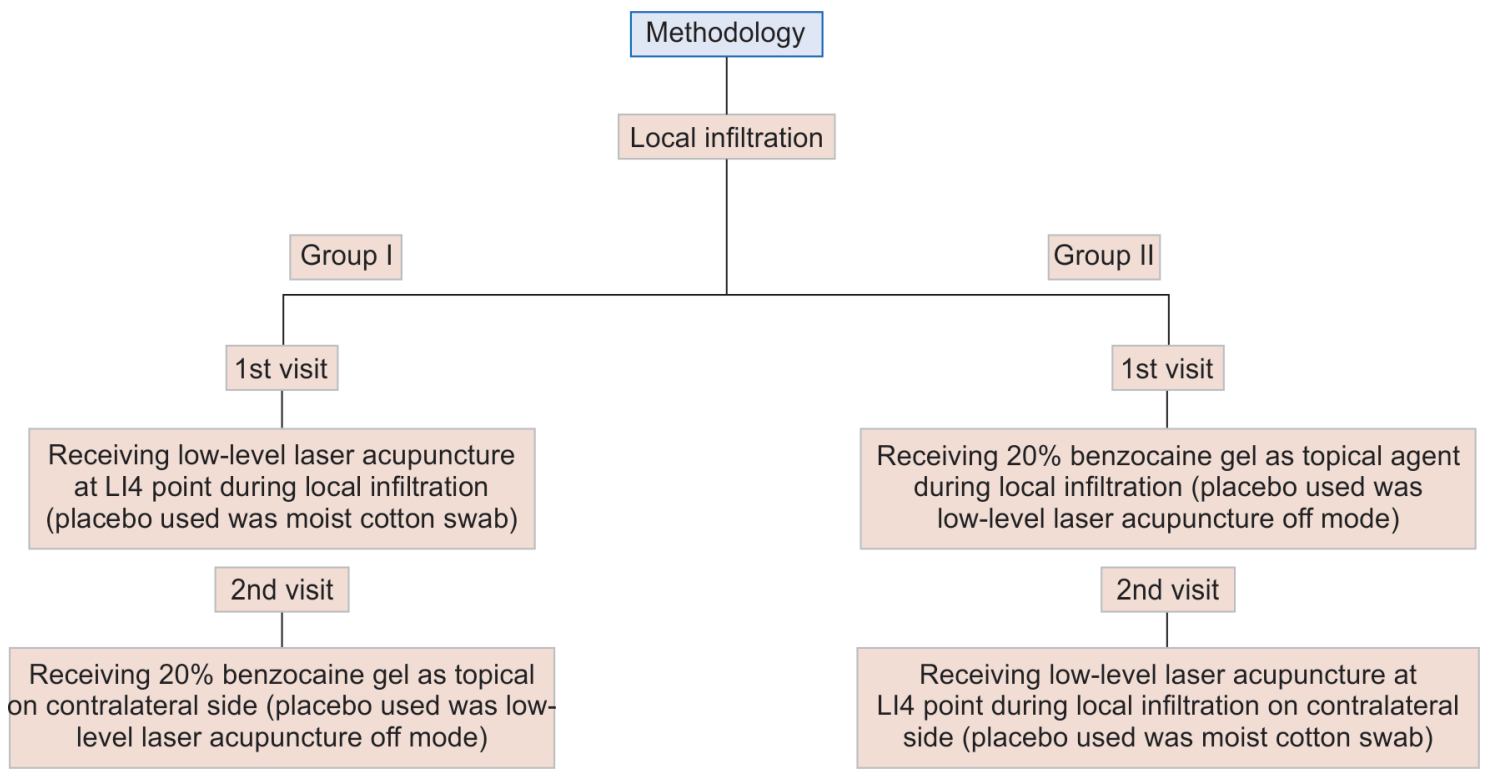


lignocaine and 1:80,000 adrenaline) of $0.5 \mathrm{~mL}$ for 30 seconds on the buccal surface of respective tooth was be given by an operator. The pulse of the patient will be examined and noted before during and after anesthesia by using a pulse oximeter.

Pain perception during local anesthesia was evaluated by the Sound, Eye, Motor (SEM) Scale as an objective scale assessed by another operator during infiltration and Wong-Baker faces pain rating scale was used as a subjective scale which was assessed by the patient. $^{15}$

\section{During the Second Visit}

In group I $(n=20)$, a cotton roll for 30 seconds was used to keep mucosa dry followed by application of $20 \%$ of topical benzocaine gel for 1 minute, ${ }^{14}$ then low-level laser acupuncture was done on "off mode" at LI4 point to induce placebo effect followed by local infiltration ( $2 \%$ lignocaine and 1:80,000 adrenaline) of $0.5 \mathrm{~mL}$ for 30 seconds on the buccal surface of respective tooth was be given by an operator. The pulse of the patient will be examined and noted before during and after anesthesia by using a pulse oximeter.

Similarly, in group II $(n=20)$, during the second visit, during the first visit, a moist cotton swab was applied in the area of infiltration to induce placebo and waited for 1 minute and then low-level laser acupuncture was done on LI4 point with a power output of $200 \mathrm{~mW}$, at a wavelength of $980 \mathrm{~nm}$, and energy of 3-4 J (Zolar photon plus diode laser) for 1 minute followed by local infiltration (2\% lignocaine and 1:80,000 adrenaline) of $0.5 \mathrm{~mL}$ for 30 seconds on the buccal surface of the respective tooth which was given by another operator who was not aware of groups ${ }^{12,13}$ (Fig. 1).

During the laser acupuncture procedure, protective eyewear was provided to the patient, operator, and assistant. After the respective local anesthesia procedure, the necessary treatment was conducted on the respective tooth.

\section{Statistical Analysis}

Data obtained were compiled on an MS Office Excel Sheet and were subjected to statistical analysis using the Statistical Package for Social Sciences (SPSS v 21.0, IBM).

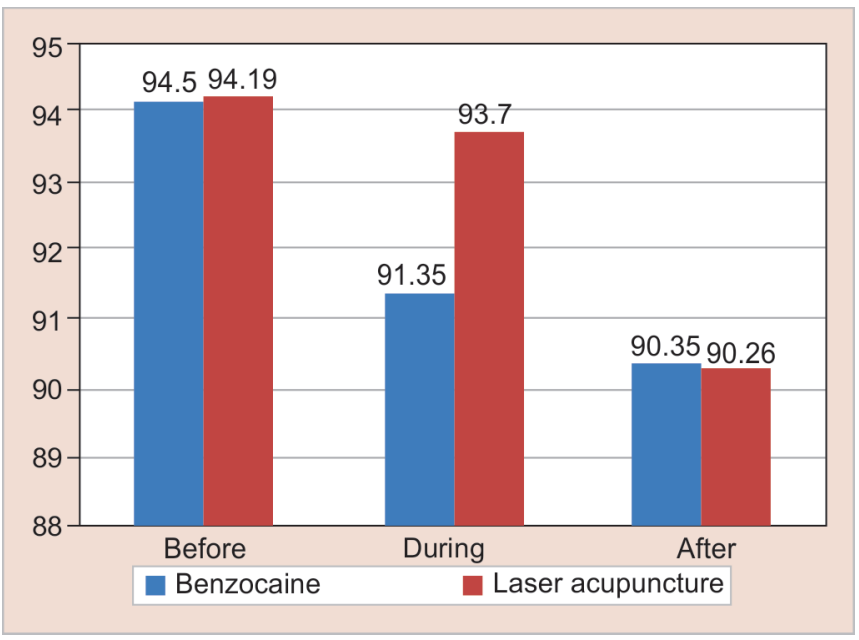

Fig. 2: Mean heart rate
Changes in heart rate were compared in-between groups using repeated measure ANOVA and intragroup comparison using independent $t$-test. For comparison of SEM and WBPRS, MannWhitney $U$ test was used of both groups depending on their mean ranks. For all the statistical tests, $p<0.05$ was considered to be statistically significant.

\section{Results}

A total number of 40 children participated and a randomized cross-over study was planned and allocated between two groups and their respective visits, i.e., in visit 1; group I $(n=20)$ : children receiving low-level laser acupuncture at LI4 point, with placebo as a moist cotton swab. Group II $(n=20)$ : children receiving $20 \%$ benzocaine gel in the area of infiltration, placebo used was lowlevel laser acupuncture on-off mode at LI4 point. Similarly, in visit 2 , group I $(n=20)$ : children receiving $20 \%$ benzocaine gel in the area of infiltration, placebo used was low-level laser acupuncture on-off mode at LI4 point and in group II $(n=20)$, children receiving low-level laser acupuncture at LI4 point, with placebo as a moist cotton swab.

Procedures were carried out as per the groups and results for heart rate, SEM, and WBPRS were obtained, were entered into a standardized form, and were statistically analyzed using SPSS version 21.0.

Mean heart rates were significantly lower among children in group I than among those in group II during the first visit (during intervention), and also in the second visit, the mean heart rate of group II showed significantly lower than group I (during intervention). Accordingly, heart rate mean scores during the first visit (before and during intervention) showed no significant differences in both groups (Figs 2 and 3).

Differences in sound eye motor scale (SCM) scale score were significantly lower in group I when compared with group II first visit (during intervention). And during the second visit SCM scale, group II showed significantly lower when compared with group I during an intervention (Fig. 4).

In Wong-Bakers pain rating scale showed significantly lower in group I when compared with group II first visit (during

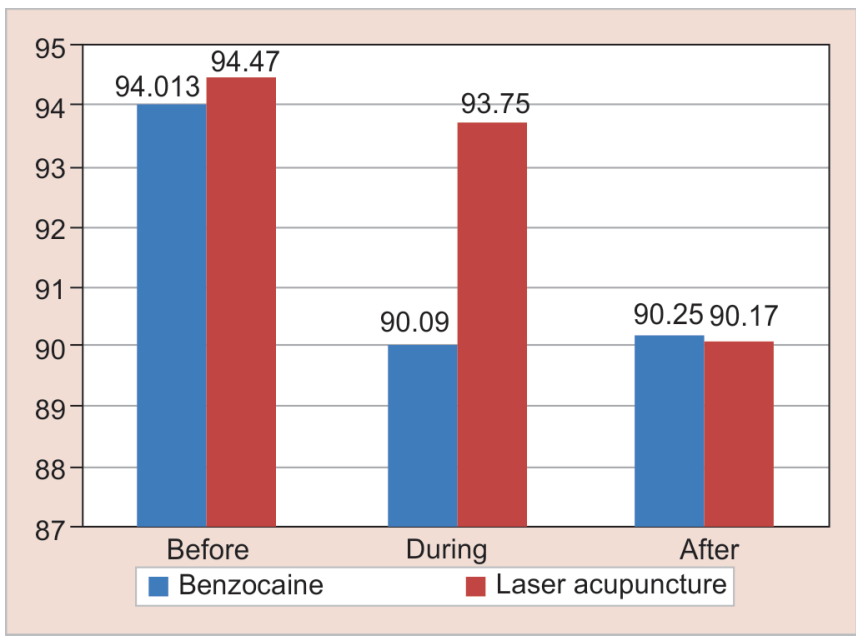

Fig. 3: Mean heart rate 


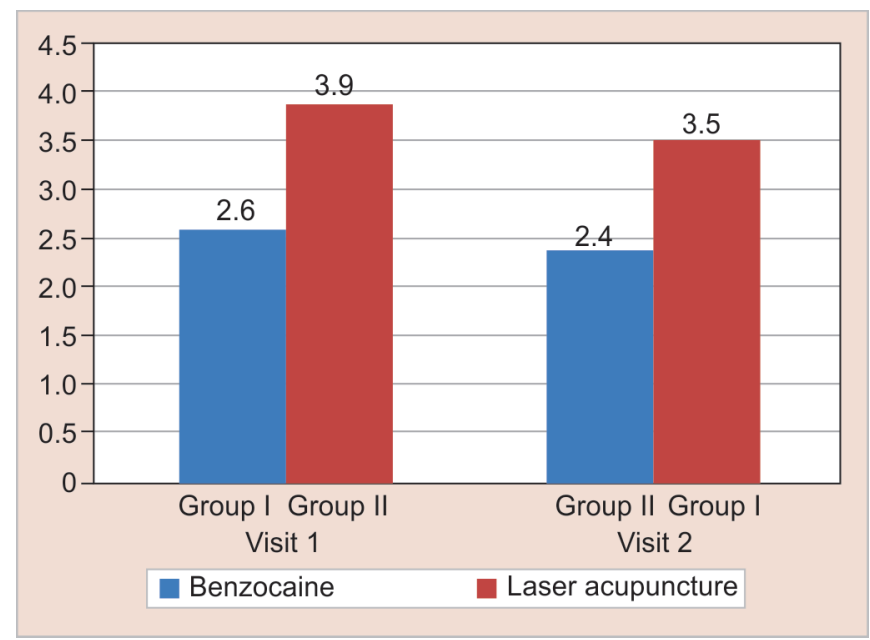

Fig. 4: Sound eye motor scale

intervention), respectively, and during the second visit group II showed significantly lower when compared with group I (Fig. 5).

In individual groups, in group l, children during the first visit showed significantly lower heart rate, SCM, and WBPRS during the intervention when compared with the second visit, similarly, in group II, during the second visit showed a significantly lower heart rate, SCM, WBPRS when compared with the first visit.

\section{Discussion}

Control of pain along with the cooperation of the child has always been a challenge when it comes to pediatric dentistry. Various methods are available to control pain during local anesthesia, like topical acting gels and sprays. ${ }^{16}$

Benzocaine is rapidly absorbed on the mucosal membrane. It is less soluble in water and has a long-acting effect with less toxicity. It crosses the oral mucosal membrane and produces analgesia and blocks the conduction of signals from the terminal fibers of the sensory nerves and producing surface anesthesia up to a depth of 2-3 $\mathrm{mm}$. It also causes alteration in transmission through voltagesensitive sodium channels, resulting in minimizing needle insertion pain effectively. ${ }^{16}$

Traditional Chinese medicine has focused on the reduction of pain using acupuncture. Acupuncture is considered a key component in traditional Chinese medicine, which has been practiced for over 25,000 years and is considered as one of the oldest healing practices in the world. ${ }^{17,18}$

According to the traditional Chinese theories, energy flows through the human body called Qi (vital force) which can be stimulated to create balance and health. This energy moves through the body along many channels known as meridians. These meridians are invisible and composed of 14 in number, they represent major organs and their functions; however, they do not follow the exact path of nerves or blood supply. These theories are similar to the concept of the "theory of gate control pain pathway". 8,17

Acupuncture point activates, stimulates A-delta and c-fiber, and sends signals to the spinal cord with the release of dynorphins and enkephalins, when these signals reach the mid-brain the excitatory and inhibitory mediators are activated and neurotransmitters are produced causing pre- and post-synaptic inhibition of pain transmission. ${ }^{17}$

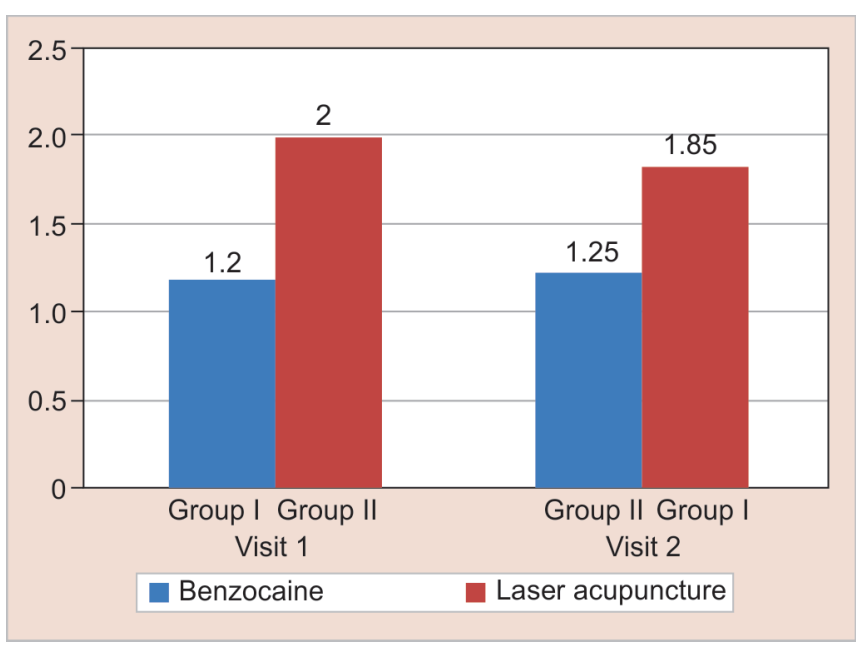

Fig. 5: Wong-Bakers pain rating scale

In the present study, LI4 acupoint or Hegu point was selected as it has been considered an analgesic effect in dental and maxillofacial structures. $^{8,9}$

Acupuncture has been used in dentistry to control pain, in a study by Usichenko et al. used bilateral acupuncture technique at LI4 using indwelling fixed needles to study the effectiveness of LI4 stimulation and concluded that the patient's heart rate was low throughout the dental treatment after LI4 stimulation when compared with treatment without acupuncture, thus proving its effectiveness. ${ }^{19}$

Also, Usichenko et al. had also conducted similar studies to see the effectiveness of LI4 point stimulation, they used "New Pyonex" needles for activation and concluded that LI4 stimulation was safe and had reduced pain during the treatment among children and parents. ${ }^{19}$

A study conducted by Jalali et al. in which needles were inserted at LI4 point and inferior alveolar nerve block was given and endodontic treatment was continued and concluded that application of acupuncture point before any endodontic treatment increased the effectiveness of IANB for mandibular molars. ${ }^{11}$

Stimulation of acupuncture points can be done by various methods using mechanical stimulation, using needles, electroacupuncture. Nowadays Chinese medicine has begun using nonthermal low-intensity lasers for stimulation of acupuncture points; metallic needles were more effective in stimulation of acupuncture points; however, they are not widely used these days due to fear of needles usage in children. ${ }^{10}$

Activation and stimulation of acupuncture points using low power laser are painless, noninvasive, atraumatic, and can be easily performed in areas having difficult access.

Few studies have been proven for the effectiveness of laser acupuncture in dentistry, it has been widely in temporomandibular joint dysfunction, myofascial pain, and gagging reflex. ${ }^{10}$

A study by Goel et al. (2017) conducted laser acupuncture at P6 acupoint for control of gagging reflex and concluded that stimulation of acupoint by low-level laser was very effective in the reduction of gag reflex..$^{15}$ A case report presented by Sarabadani et al. (2018) studied control of gag reflex by stimulation of various acupuncture points using low-level laser therapy during composite restorations and concluded that low-level laser therapy 
was effective in the control of gag reflex. A similar wavelength of $980 \mathrm{~nm}$ and $200 \mathrm{~mW}$ was used in the present study. ${ }^{13}$

So, a low-level laser was used at LI4 point giving a good stimulation effect as compared to the study conducted by Goel et al. and Sarbandani et al.

The main advantage of using a low-level laser than manual needling technique is that it requires fewer clinical appointments, that is, 20 seconds to 15 minutes and needle acupuncture takes about 15-30 minutes for stimulation. ${ }^{20}$

Thus, with the reference to the results of this study, low-level laser therapy can be used to control pain during local anesthesia injection in dentistry.

\section{Conclusion}

Low-level laser acupuncture has demonstrated analgesic effects and it can be used to control pain during the local anesthetic procedure in pediatric patients when compared with $20 \%$ benzocaine gel. However, further studies have to be conducted using a higher sample size.

\section{References}

1. McDonald RE, Avery DR, Dean JA, ed. Local anesthesia and pain control for the child and adolescent. In: Dentistry for the child and adolescent. 8th ed., St. Louis, Mo: CV Mosby Inc; 2004. p. 272.

2. Milgrom $P$, Mancl L, King B, et al. Origins of childhood dental fear. Behav Res Ther 1995;33(3):313-319. DOI: 10.1016/0005-7967(94)00042i.

3. Vika M, Raadal M, Skaret $E$, et al. Dental and medical injections: prevalence of self-reported problems among 18 -yr old subjects in Norway. Eur J Oral Sci 2006;114(2):122-127. DOI: 10.1111/j.16000722.2006.00335.x.

4. Ram D, Peretz B. The assessment of pain sensation during local anesthesia using a computerized local anesthesia (Wand) and a conventional syringe. J Dentis Child 2003;70(2):130-133.

5. Kreider KA, Stratmann RG, Milano M, et al. Reducing children's injection pain: lidocaine patches versus topical benzocaine gel. Pediat Dentis 2001;23(1):19-23.

6. World Health Organization. Acupuncture: review and analysis of reports on controlled clinical trials. Geneva: World Health Organization; 2003. p. 6.
7. Wong LB. Acupuncture in dentistry: its possible role and application. Proc Sing Healthc 2012;21(1):48-56. DOI: 10.1177/2010105812021 00108.

8. Liu J, Ni L. Acupuncture in dentistry. J N J Dent Assoc 1974;45:16-18.

9. Wang SM, Kain ZN, White PF. Acupuncture analgesia: II. Clinical considerations. Anesth Analg 2008;106(2):611-621. DOI: 10.1213/ ane.0b013e318160644d.

10. Ferreira LA, Oliveira RG, Guimarães JP, et al. Laser acupuncture in patients with temporomandibular dysfunction: a randomized controlled trial. Lasers Med Sci 2013;28(6):1549-1558. DOI: 10.1007/ s10103-013-1273-x.

11. Jalali S, Majd NM, Torabi S, et al. The effect of acupuncture on the success of inferior alveolar nerve block for teeth with symptomatic irreversible pulpitis: a triple-blind randomized clinical trial. J Endod 2015;41(9):1397-1402. DOI: 10.1016/j.joen.2015.04.026.

12. Massir E, Palmon Y, Zilberman U. Local anesthesia in pediatric dentistry-how much is enough. Dentistry 2018;8:480. DOI: 10.4172/2161-1122.1000480.

13. Sarabadani J, Labafchi A, Chiniforush N. Controlling gag reflex whit low power laser acupuncture on point P6: a case report. Regenerat Reconst Restorat 2018;3(3).

14. Garg A, Garg N, Kaur D, et al. Evaluation of efficacy of 2\% lidocaine gel and $20 \%$ benzocaine gel for topical anesthesia. Endodontology 2016;28(1):38. DOI: 10.4103/0970-7212.184338.

15. Goel H, Mathur S, Sandhu M, et al. Effect of low-level LASER therapy on P6 acupoint to control gag reflex in children: a clinical trial. J Acupunc Mer Stud 2017;10(5):317-323. DOI: 10.1016/j.jams.2017.07.002.

16. Nair M, Gurunathan D. Comparative evaluation of the efficacy of two anesthetic gels ( $2 \%$ lignocaine and $20 \%$ benzocaine) in reducing pain during administration of local anesthesia-a randomized controlled trial. J Anaesthes, Clin Pharma 2019;35(1):65. DOI: 10.4103/joacp. JOACP_73_18.

17. Katti G, Karuna CPK, Shahbaz S. Use of acupuncture as a novel practice in the management of dental diseases: a review. Int J Scienti Study 2014;2(6):112-117.

18. Plog FMW. Biophysical application of the laser beam. In: Koebner HK, ed. Lasers Med. Chichester: Wiley; 1980. pp. 21-37.

19. Usichenko TI, Wolters $P$, Anders EF, et al. Acupuncture reduces pain and autonomic distress during injection of local anesthetic in children. Clin J Pain 2016;32(1):82-86. DOI: 10.1097/AJP.0000000000000222.

20. de Oliveira RF, da Silva CV, Cersosimo MC, et al. Laser therapy on points of acupuncture: are there benefits in dentistry? J Photochem Photobiol B: Biol 2015;151:76-82. DOI: 10.1016/ j.jphotobiol.2015.07.008. 